\title{
Modeling and simulation of signal transductions in an apoptosis pathway by using timed Petri nets
}

\author{
Chen Li* Qi-Wei GE ${ }^{\dagger} \quad$ Mitsuru Nakata ${ }^{\dagger}$ \\ Hiroshi Matsuno* Satoru Miyano ${ }^{\S}$ \\ ${ }^{*}$ Graduate School of Science and Engineering, ${ }^{\dagger}$ Faculty of Education, Yamaguchi University, \\ 1677-1 Yoshida, Yamaguchi 753-8511, Japan \\ ${ }^{\S}$ Human Genome Center, Institute of Medical Science, University of Tokyo, 4-6-1 Shirokane-dai, \\ Minato-ku, Tokyo 108-8639, Japan \\ ${ }^{\S}$ Corresponding author (Fax, 81-3-5449-5442; Email, miyano@ims.u-tokyo.ac.jp)
}

\begin{abstract}
This paper first presents basic Petri net components representing molecular interactions and mechanisms of signaling pathways, and introduces a method to construct a Petri net model of a signaling pathway with these components. Then a simulation method of determining the delay time of transitions of Petri net model, i.e. the time taken in firing of each transition, is proposed based on some simple principles that the number of tokens flowed into a place is equivalent to the number of tokens flowed out. Finally, the availability of proposed method is confirmed by observing signaling transductions in biological pathways through simulation experiments of the apoptosis signaling pathways as an example.
\end{abstract}

Keywords. Signaling pathway; Petri net; modeling; simulation; apoptosis

\section{Introduction}

Systems biology is a new field that aims to integrate different levels of information to understand how biological systems function. Generally in systems biology, mathematical modeling, simulation and analysis of biological systems play a critical role in helping biologists and biochemists explain and predict system's behavior.

Petri net is a formal description for modeling concurrent systems [22], and recently have been widely accepted as a description method for biological pathways by researchers in computer science as well as those in biochemistry [24]. Various types of Petri net (e.g. low level Petri nets [5, 6, 26, 27], stochastic Petri nets [20, 21], hybrid Petri nets [16, 17], colored Petri nets [3]) have been widely applied to study metabolic pathways and signaling pathways in both quantitative and qualitative approaches because of potential advantages of Petri net possessing intuitive graphical representation and capabilities for mathematical analysis.

Metabolic pathways have such intrinsic characteristic that are series of chemical reactions catalyzed by enzymes, resulting in either the formation of a metabolic product to be used or stored by the cell, or the initiation of other metabolic pathways. The idea to use Petri nets for modeling and simulating metabolic pathways have been popularly developed $[8,11,26,27,31,34,37]$ owing to the simple mechanism that can be expressed by a uniform network of catalytic reactions.

In contrast, signaling pathways are generally more complex, consisting of distinct reactions such as complex formation, catalytic reaction, and translocation. With the feature of signaling pathways, a few researchers have tried to investigate relationships among complex molecular mechanisms and interactions, and further structural behaviors of signaling pathways by using Petri nets [1, 6, 12]. In the paper [6], Heiner et al. have explained how to model and validate the apoptosis pathways by using qualitative Petri nets. They have demonstrated a step-wise technique to model apoptosis signaling pathways. Further they have performed the model validation by using a standard Petri net analysis technique, and presented the biological meaning of the analysis results. Nevertheless, it is still expected to find a general methodology to model and analyze common signaling pathways by using Petri nets.

The current standard approach to represent biochemical reactions as a system is to use a series ordinary differential equations (ODEs). This approach provides mathematically well-founded and fine interpretations of biological pathways. Accordingly, some trials have been made to model and simulate signaling pathways using ODEs $[4,29]$. Though ODEbased simulation can present quantitative behaviors of biological substances, it is hard to observe the whole system intuitively and grasp structural images of biological pathways from the series of differential equations. One approach to cope with this problem is to use hybrid Petri net (HPN) $[15,16]$ which allows quantitative modeling and simulation of biological pathways with taking advantages of Petri net enabling graphical representation of biological pathways.

Quantitative analyses have also been made using discrete Petri net as found in the papers $[3,8,25]$. Popova-Zeugmann et al. [25] have introduced time Petri nets to bridge the gap between qualitative and quantitative models in steady state. They have presented structural techniques to decide the timedependent realizability of a given transition sequence and to 
calculate its shortest and longest time length for the analysis of the time Petri net model.

In this paper, we propose a method of determining the delay time of transitions for firing by using timed Petri net models and perform simulations without establishing and tuning exact concentration. The paper is organized as follows. Firstly, we present a brief introduction of Petri net. Then we introduce a modeling method based on Petri net by taking notice of molecular interactions and mechanisms, and propose rules to determine the delay time of transitions. Further we show the Petri net model after explaining the biological background of Fas-induced apoptosis as an example. Finally, we use the example to demonstrate the usefulness of our modeling and simulation method by using a Petri net based simulation tool "Cell Illustrator" [2].

\section{Modeling signaling pathways with Petri nets}

Petri nets are powerful tools in modeling and simulating various concurrent systems [22], especially biological pathways because Petri nets have the following superior characteristics [18]:

(1) "firm mathematical foundation" enabling formal and clear description of biological pathways as well as their structural analysis, and

(2) "visual representation of networks" which provides intuitive understanding of biological pathways without any mathematical descriptions which are basically difficult for ordinary biologists.

In this section, we give the modeling rules for signaling pathways based on Petri net representation. The aims of the modeling by Petri net for signaling pathways are: (i) to make the biologists intuitively understand the intrinsic structure and features of signaling pathways and (ii) to make it possible to mechanically model larger and more complicated signaling pathway networks.

\subsection{Basic definitions}

A Petri net $N$ is defined by a 5 -tuple $N=(T, P, E, \alpha, \beta)$ that corresponds to a bipartite graph, where $T$ is a set of transitions represented by bars or boxes, $P$ is a set of places represented by circles in a graph, $E$ is a set of directed arcs between places and transitions, $\alpha$ denotes the weight of arc from place to transition, and $\beta$ denotes the arc weight connected from transition to place. Note that the presence of multiple arcs is shown by a single arc with a non-zero positive integer arc weight.

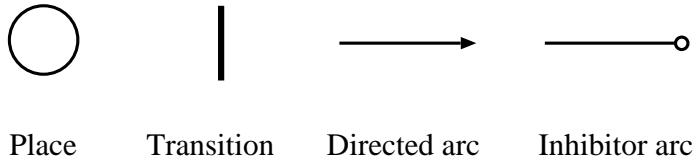

Figure 1: Basic elements of Petri net.

A place can hold a positive integer number of tokens as its content. An assignment of tokens in each place expressed in form of a vector is called marking $M$, which varies during execution of a Petri net. A transition without input places is

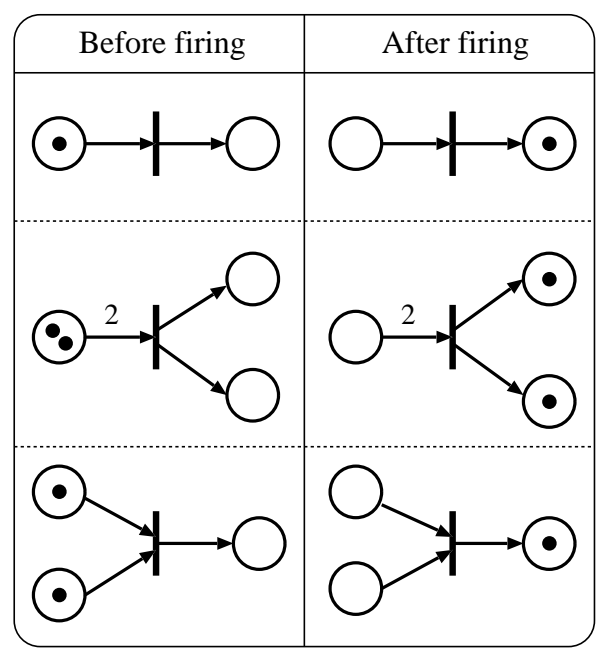

Figure 2: Examples show firing rules of Petri net.

called source transition that is always firable, and a transition without output places is called a sink transition likewise.

[Firing rule of Petri nets $N$ ] A transition $t$ is firable if each its input place $p_{I}$ has at least $\alpha_{e}\left(e=\left(p_{I}, t\right)\right)$ tokens. A transition $t$ fires to remove $\alpha_{e}\left(e=\left(p_{I}, t\right)\right)$ tokens from each its input place $p_{I}$ and deposit $\beta_{e}\left(e=\left(t, p_{O}\right)\right)$ tokens to each its output place $p_{O}$.

An inhibitor arc represents inhibitory function which is depicted as a line with a hollow circle at the end where the arrowhead normally appears. An inhibitor arc disables a transition to fire if the upstream place is occupied by a token, but does not consume the token. Figures 1 and 2 show basic elements of Petri net and the transition firing rules, respectively. For the details of Petri net theory, the readers are suggested to refer to Ref. [22].

To do simulation of systems, Petri net model is usually extended by assigning firing time delay to transitions. Such extended Petri net is called timed Petri net $\bar{N}=(\bar{T}, \bar{P}, \bar{E}, \alpha, \beta)$. The firing rule of timed Petri net is extended as follows:

[Firing rule of timed Petri nets $\bar{N}$ ] (1) If the firing of a transition $t_{i}$ is decided, tokens required for the firing are reserved. (2) When the delay time $d_{i}$ of transition $t_{i}$ passed, $t_{i}$ fires to remove the reserved tokens from the input place of $t_{i}$ and put tokens into the output places of $t_{i}$.

In a timed Petri net, the firing times per unit time $f_{i}$, called firing frequency, of a transition $t_{i}$ is constrained by its delay time $d_{i}$, and the maximum of firing frequency is the reciprocal of $d_{i}$.

\subsection{Modeling rules}

Here, we give the modeling method for signaling pathways with Petri net that can be naturally and explicitly modeled according to the following rules:

(1) Places denote static elements including chemical compounds, conditions, states, substances and cellular organelles participating in the biological pathways. Tokens indicate the presence of these elements. The number of tokens is given to represent the amount of chemical substances. 


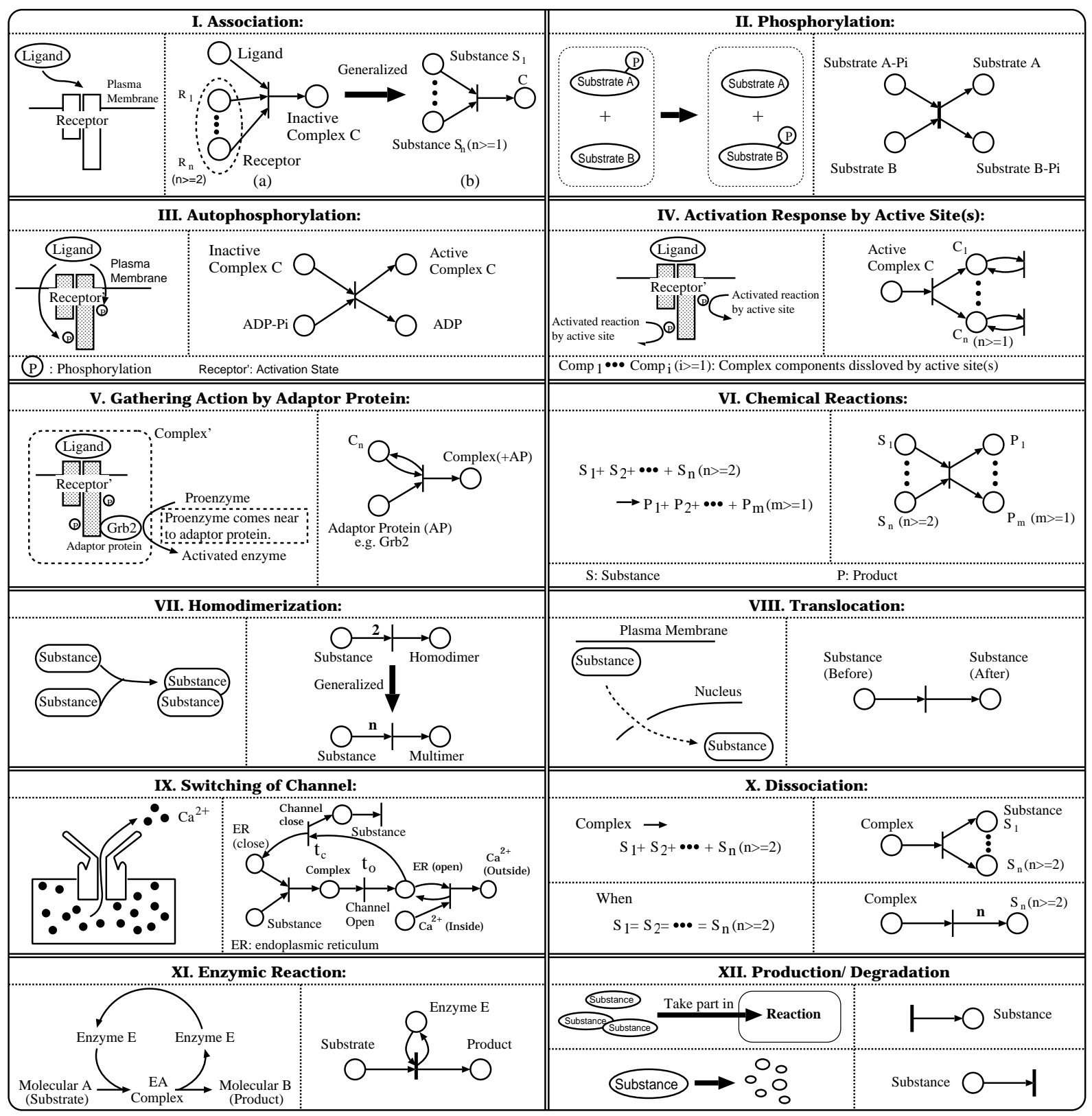

Figure 3: Petri net models of various reaction types in signaling pathways.

(2) Transitions denote active elements including chemical reactions, events, actions, conversions and catalyzed reactions. A transition fires by taking off tokens from its individual input places and creating new tokens that are distributed to its output places if its input places has at least as many tokens in it as arc weight from the place to the transition.

(3) Directed arcs connecting the places and the transitions represent the relations between corresponding static elements and active elements. Arc weights $\alpha$ and $\beta$ describe the quantities of substances required before and after reaction, respectively. Especially in case of modeling a chemical reaction, arc weights represent quantities given by stoichiometric equations of the reaction itself. Note that, weight of an arc is omitted if the weight is 1 .

(4) An inhibition function in biological pathways is modeled by an inhibitor arc.
In cell biology, signaling pathways have been widely studied. They are information cascades of enzyme reactions from transmembrane receptors to the nucleus DNA, which ultimately regulate intracellular responses such as programmed cellular proliferation, gene expression, differentiation, secretion and apoptosis. Numerous reaction types of molecular interaction mechanisms have been described by Petri net model [7], which suffices to give the description of the metabolic pathway presently [26]. For signaling pathways, as has been pointed [32], besides the catalytic reactions, the information among the molecular interactions such as complex formation, gathering action, translocation and channel switching, also need to be respectively modeled by Petri nets according to different types of interactions as long as the biological facts has been known.

To explicitly understand the structural complicated signaling pathways, the modeling of each essential molecular interaction by using Petri net is the first step in modeling the 
network of signaling pathways as a qualitative event system. With a focus on possible molecular interactions as long as we have known, we summarize various molecular interactions of signaling pathways (see left side of dashed line in figure 3) and their corresponding Petri net model (right side of dashed line). Both of them in a reaction type are described as a "block" labeled with roman numeral in this paper. The description of each molecular interaction and corresponding model are given as follows:

I. Association reaction is a binding reaction to induce the formation of homo- or heterodimers and to generate a complex compound. This block shows the ligand-receptor binding interaction and corresponding Petri net model that indicates the transition is unfirable in the absence of place of ligand although receptors exist. The number of input place of transitions is two or more while the output place number is one in association reaction. Obviously, we also can expand the conception of association to the formation of model represented in block $\mathbf{I}(\mathrm{b})$, generally representing the simultaneous association of substrates $S_{1}, \cdots, S_{n}(n \geq 1)$ forming a complex $C$ in biological systems.

II. Phosphorylation is a reaction to add a phosphate $\left(\mathrm{PO}_{4}\right)$ group to a protein or a small molecule, and dephosphorylation that is the backward reaction of phosphorylation removing phosphate groups from a compound by hydrolysis.

III. Autophosphorylation is a transphosphorylation reaction frequently following the binding of a ligand to a receptor with intrinsic protein kinase activity.

IV. Generally continued activated ligand-receptor complex regulates varied majority of cellular pathways transmitting the signals within the cell. Few methods using Petri nets have been proposed to model such activated complex place possessing more than one transitions that can trigger downstream signaling pathways [6]. Their methods are easily understood, but have some problems that, if the transition of such place fires to remove the token(s) in shared input place at one time epoch, it will disable rest transitions simultaneously although the token will return back the same input via a self-loop. Hence, we need a more appropriate model to express this system's behavior. Our basic consideration is that, if there have plurality of successive signaling pathways depending on distinct active site(s) (subunits) of activated complex, all the active site(s) shall be regarded as complex component(s) $C_{1}, \cdots, C_{n}(n \geq 1)$ as shown in block $\mathbf{~ I V}$.

V. Gathering action by adaptor protein is distinguished from association reaction. The main participator adaptor protein is an accessory protein to main proteins. These proteins lack the intrinsic enzymic activities themselves but instead mediate specific protein-protein interactions driving the formation of protein complexes.

VI. Chemical reactions is the most common reaction in signaling pathways, which the conversion of substances to products is ordinarily modeled as input places to output places, both belonging to the same transition.

VII. Homodimerization is a polymerization reaction of two identical substances to shape a dimmer similar to a kind of association reaction. A substance is modeled as an input place connected with a 2-weighted arc. It is easy to expand the conception to model the formation of multimer holding $n$-weight such as trimer and tetramer that is a complex of two or more equivalent polypeptides. The lower Petri net in block $\mathbf{X}$ is constructed under the opposite consideration of modeling homodimerization reactions.

VIII. Translocation refers to the movement of molecules, substances or ions across cell membranes or via the bloodstream in biology. Figure 3 shows the nuclear translocation within a cell. A transition is modeled to indicate the movement action of substances before and after.

IX. Intracellular signal pathways are largely carried out by second messenger molecules. $\mathrm{Ca}^{2+}$ acts as a second messenger molecule to carry out large intracellular signal inside the cell. Usually the concentration of free $\mathrm{Ca}^{2+}$ within the cell is very low; it is stored inside of organelles, mostly the endoplasmic reticulum. In order to become active, $\mathrm{Ca}^{2+}$ has to be released from the organelles into the cytosol. Two transitions $t_{o}$ and $t_{c}$ are introduced to denote channel activity of "open" and "close", respectively. $t_{o}$ is enabled when input place holds up token(s) after the association of organelles and substances, whereas $t_{c}$ is enabled as long as some stop mechanisms shutoff the channel.

$\mathbf{X}$. This is the opposite of $\mathbf{I}$. Dissociation process is a general process in which complexes and molecules separate or split into smaller molecules, ions. The number of input place of transitions is one while the output place number is two or more.

XI. Since an enzyme itself plays a role of catalyzer in biological pathways and there occurs no consumption in biochemical reactions, the reaction is modeled to a transition, whereas the substrate is modeled to enzyme place that has a self-loop with same arc-weight. That is, once an enzyme place is occupied by a token, the token will return to the place again to keep the firable state, if the transition is fired.

XII. A source transition represents an activity to provide substances that will take part in the reactions. A sink transition denotes small and natural degradation of substance.

\section{Modeling and simulation of signaling pathways}

In this section, we use the example of apoptosis to demonstrate our modeling method and then explain how to simulate.

\subsection{Biology background and modeling of apoptosis}

Apoptosis, a form of cell death characterized by cell shrinkage, membrane blebbing, nuclear breakdown, and DNA fragmentation, is a vital cell lifecycle decision point for development, maintenance of tissue homeostasis, and elimination of harmful cells in metazoan organisms $[10,19]$. Caspases (cysteine-aspartic-acid-proteases) are a group of cysteine proteases existing as inactive zymogens that can be cleaved by other proteins within the cell resulting in the apoptotic process. Failure of apoptosis is one of the main contributions to tumor development and autoimmune diseases such as neurodegenerative diseases, AIDS and ischemic stroke [33]. Different cellular signals can initiate activation of apoptosis on different ways in dependence of the various kinds and biological states of cells. Fas-induced apoptosis has been studied in detail and its mechanism has been proposed. Fas ligand is a type II transmembrane protein belonging to TNF fam- 


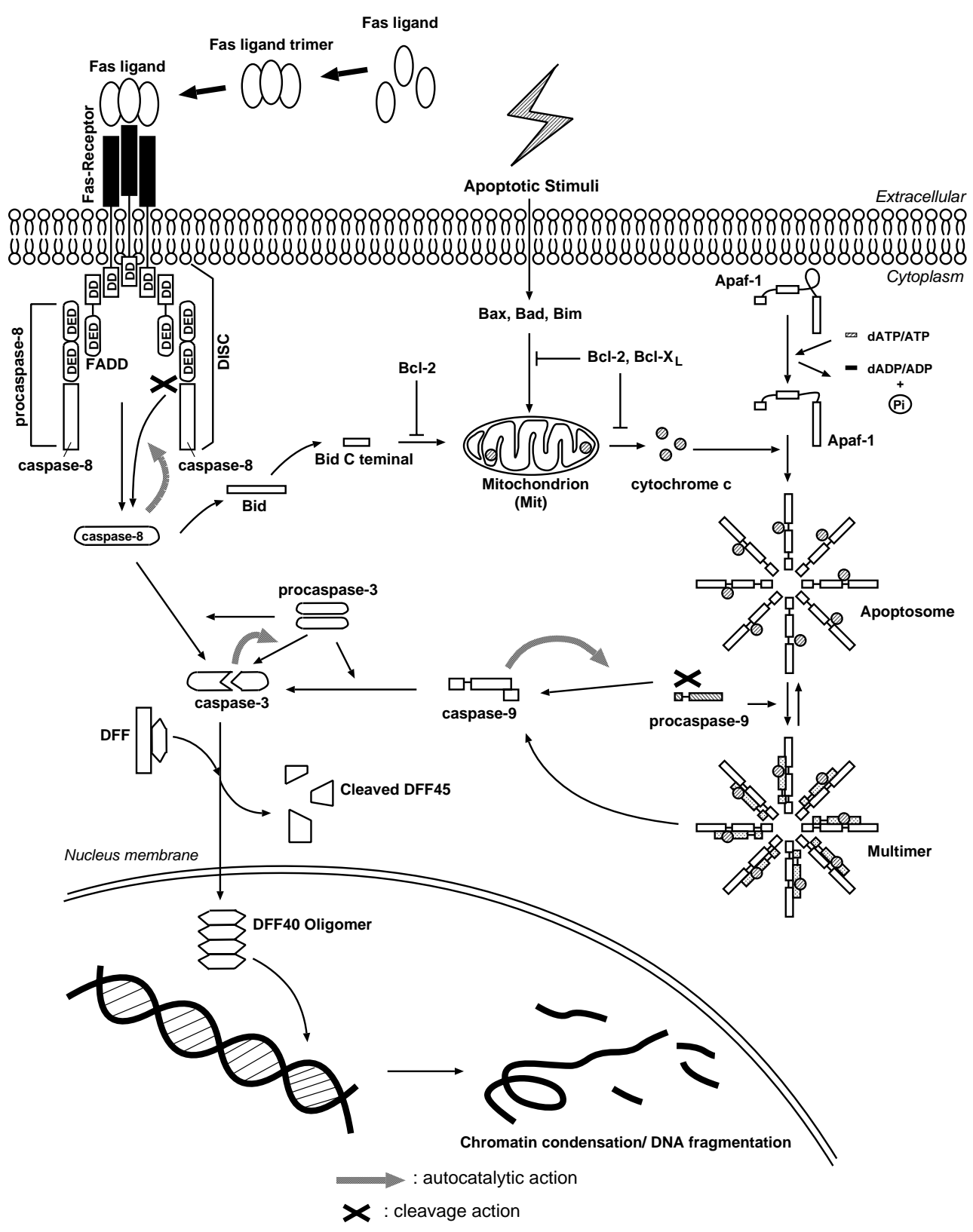

Figure 4: Biological diagram of apoptosis pathways induced by Fas ligands.

ily, which signals apoptotic effects to nucleus through several major pathways as shown in figure 4 .

Fas ligands, existing as trimers, bind to Fas receptor and promote receptor trimerization. Adaptor proteins FADD in turn associate with the receptors through an interaction between homologous death domain (DD) on the receptor and FADD. Furthermore, FADD contains death effector domain (DED) that allows binding of procaspase-8 to the receptor complex to form a death inducing signaling complex (DISC) [30, 35]. Upon the association with FADD through DED, procaspase-8 is autocatalytically activated to produce caspase-8. The activation of caspase- 8 initiates the following two pathways (1) and (2) leading to the activation of downstream caspases.

(1) caspase-8 activates downstream caspases indirectly by cleaving $\mathrm{Bcl}-2$ interacting protein (Bid) and $\mathrm{COOH}$-terminal part of Bid (tBid) translocates onto mitochondria (Mit for short) where it makes cytochrome c leak out and enter the cytosol. Released cytochrome $\mathrm{c}$ binds to apoplectic protease activating factor-1 (Apaf-1) together with dATP/ATP and procaspase- 9 and achieve the caspase- 9 activation based on three-step reactions as illustrated in figure $4[9,13,38]$ :

(i) dATP/ATP binds to Apaf-1 and is hydrolyzed to dADP or ADP, respectively;

(ii) cytochrome $\mathrm{c}$ binds to Apaf-1 and promotes the multimerization of Apaf-1/cytochrome c complex, forming a so-called apoptosome made of at least eight subunits when dATP/ATP bound to Apaf-1 is hydrolyzed;

(iii) Once apoptosome is formed, procaspase-9 is recruited to apoptosome, and it becomes activated through autocatalysis.

Activated caspase-9 releases from apoptosome to cleave downstream caspases such as caspase-3, and new procaspase- 9 is activated through autocatalysis by either apoptosome or fresh activated caspase-9 [14]. 


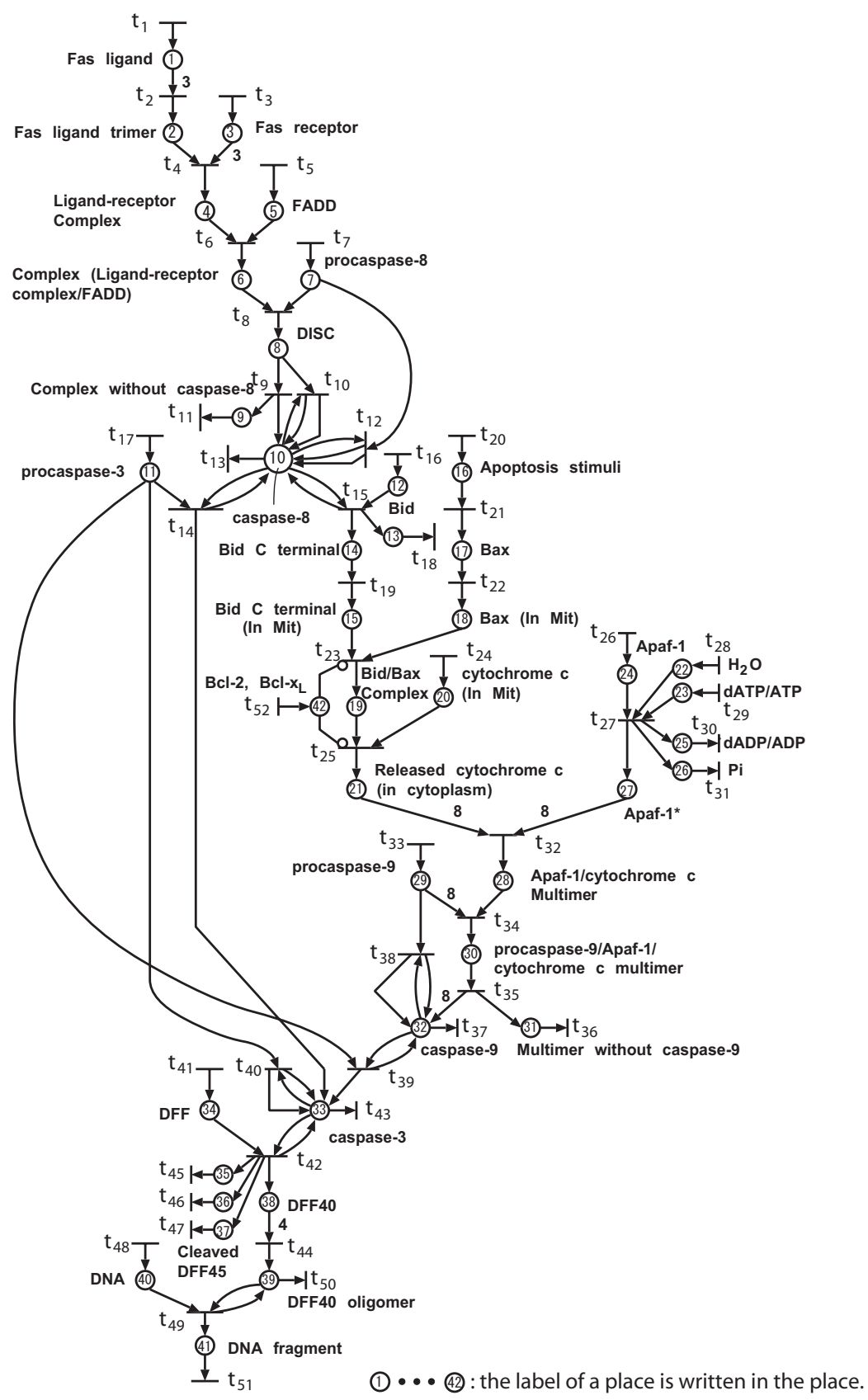

Figure 5: Petri net model without time of figure 4.

(2) caspase-8 activates downstream caspases such as caspases- 3 by directly cleaving them.

Activated caspase-3 cleaves DNA fragmentation factor (DFF) composed of $45 \mathrm{kDa}$ (DFF45) and 40kDa (DFF40) subunits. Cleaved DFF45 dissociates from DFF40, accompanied by DFF40 homo-oligomer formation that possess DNase activity [28, 36]. Finally, DFF40 oligomer induces DNA fragmentation and chromatin condensation that is regarded as an apoptotic hallmark.

Figure 5 shows the whole Petri net model of Fas induced apoptosis based on our modeling rules. The modeling operation starts from the source transition $t_{1}$ denoting an activity that the substances take part in reactions. Here we only explain the case of the pathway whose caspases are directly cleaved by caspase-8 $\left(p_{10}\right)$ to trigger DNA damage: Fas ligands and corresponding receptor are assigned to places $\left(p_{1}\right.$ and $p_{3}$ ) connected from the source transitions $\left(t_{1}\right.$ and $\left.t_{3}\right)$ re- spectively, in accordance with block XII of figure 3. Since three Fas ligands shape a Fas ligand trimer, a transition $t_{2}$ is used to represent polymerization connecting from the input place $p_{1}$ with a 3 -weighted arc $e\left(p_{1}, t_{2}\right)$ as well as connecting to the output place $p_{2}$ of Fas ligands trimer applying the block VII in figure 3. Next, the ligand-receptor binding interaction occurs and corresponding Petri net model that two places $\left(p_{2}\right.$ and $\left.p_{3}\right)$ merge into a place $p_{4}$ denoting ligand-receptor complex via the transition $t_{4}$ represents association reaction. Succedent reactions for the production of intermediate products: ligand-receptor/FADD complex $\left(p_{6}\right)$ and DISC $\left(p_{8}\right)$ are modeled in the same way using both of block $\mathbf{I}$, i.e. association reaction. procaspase-8 $\left(p_{7}\right)$ contained in DISC $\left(p_{8}\right)$ is autocatalytically cleaved to produce caspase- $8\left(p_{10}\right)$ which is modeled as a dissociation reaction $\mathbf{X}$ represented by $t_{9}$. Since caspase-8 $\left(p_{10}\right)$ next initiates the activation of downstream caspases and there occurs no consumption in the reactions, 
caspase-8 $\left(p_{10}\right)$ is modeled to enzyme place with a self-loop to take part in four enzymic reactions including two autocatalytic activations of new caspase-8, activation of caspase-3 cascades and mitochondrial DNA damage pathways. The reaction that activated caspase-3 catalyzes DFF $\left(p_{34}\right)$ to the productions of DFF40 and DFF45, is modeled as an enzymic reaction represented by $t_{42}$. DFF40 further form a DFF40 homo-oligomer $\left(p_{39}\right)$ which applies the reaction model of homodimerization as shown in block VII represented by $t_{44}$. At last, the transition $t_{49}$ is used to model the enzymic reaction where DFF40 oligomer acts as DNase to induce DNA fragmentation whose corresponding place $p_{41}$ connects to sink transition $t_{51}$ indicating the natural degradation to the system environment (see block XI and XII).

In this way, modeling of apoptosis can be done by connecting the reaction types listed in figure 3 . The biological interpretation for each transition used in this model are summarized in table 1 . Note that this model is of no delay time for transitions.

\subsection{Creating timed Petri net for pathway simulation}

As described above, the Petri net model illustrated in figure 5 has been drawn. This model describes the structure of connection relation. The next task we should do is to confirm the validity of the model, that is whether proposed models accord with the biological facts of reactions or not. Therefore, we consider a new simulation method by which (1) the traces of signal transductions can be easily understood and (2) the transduction speeds of each pathways leading to cell death can be observed.

For controlling the transduction speeds of signals in a pathway, each transition in the Petri net model should have delay time reflecting the speed of corresponding biological reactions. Basic facts for deciding delay time can be obtained from biological experiments and scientific common principles. However, in the majority of cases, reliable data of detailed reactions have not been reported in biological literature.

These observations lead us to develop a new method that determines transition speeds with which token flows in the modeled Petri net represent the signal transductions in the original signaling pathways.

\section{[Basic principles]}

(1) The sum of consumption is equal to the production to keep the concentration equilibrium for each substance engaged in signaling pathways, i.e. for each place the token amounts flowed in and flowed out per unit time are equivalent:

$$
\sum_{i=1}^{m} K_{I_{i}}=\sum_{j=1}^{n} K_{O_{j}}
$$

$\sum_{i=1}^{m} K_{I_{i}}$ and $\sum_{j=1}^{n} K_{O_{j}}$ are the total token amounts flowed in and flowed out per unit time, respectively (see figure 6).

(2) As defined in section 2.1, delay time $d_{i}$ is the reciprocal of the maximum of firing frequency $f_{i}$, and it is obvious that the token amounts flowed in and flowed out for each place per unit time are expected to be kept equivalent under the fastest firing frequency $f_{i}$. Therefore, in this paper we decide $d_{i}$ by calculating $f_{i}$.

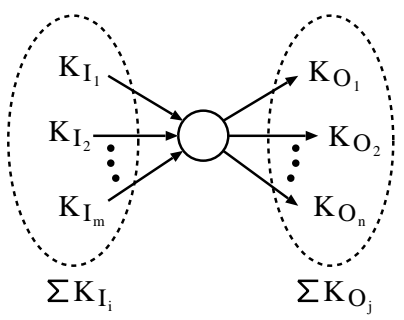

Figure 6: Illustration for [Basic principles] (1).

Note that, in this paper we suppose that apoptosis pathway is such a biological system that if required substances are assembled, the reactions promptly become possible.Thus, what we have to discuss is how to assign the delay time to each transition in timed Petri net $\bar{N}$. In the following, we give such delay time determination rules for each transition.

\section{[Strategy for determining transition speeds]}

Rule (1): if there is such a place $p_{i}$ that the number of input transitions is one or more while the number of output is one, the maximum of firing frequencies $\left\{f_{i}\right\}$ satisfy the following equation:

$$
\sum_{i=1}^{m} \beta_{i} \cdot f_{I_{i}}=\alpha \cdot f_{O}
$$

where $\alpha$ and $\beta_{i}$ are the weights of $\operatorname{arcs} e\left(p_{i}, t_{O}\right)$ and $e\left(t_{I_{i}}, p_{i}\right)$, respectively. $t_{I_{i}}$ and $t_{O}$ are the input and output transitions of place $p_{i}$. Furthermore, $f_{I_{i}}, f_{O}$ are the maximum of firing frequencies of $t_{I_{i}}, t_{O}$, respectively (see figure 7).

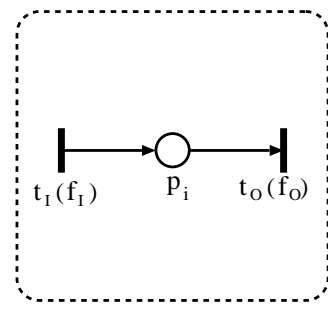

(1)

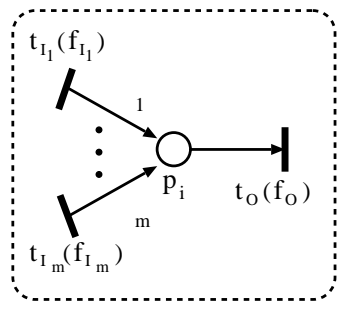

(2)
Figure 7: Two connection cases applied to Rule (1).

Rule (2): if there is a place $p_{i}$ whose output transitions are in conflict, the maximum of firing frequencies $\left\{f_{i}\right\}$ satisfy following linear equation and inequality:

$$
\left\{\begin{array}{l}
\sum_{i=1}^{m} \beta_{i} \cdot f_{I_{i}}=\sum_{j=1}^{m} \alpha_{j} \cdot f_{O_{j}} \\
2 \cdot \frac{f_{O_{n}}}{\alpha_{n}} \geq \frac{f_{O_{1}}}{\alpha_{1}} \geq \frac{f_{O_{2}}}{\alpha_{2}} \geq \cdots \geq \frac{f_{O_{n}}}{\alpha_{n}}
\end{array}\right.
$$

where $\alpha_{j}$ and $\beta_{i}$ are the weights of $e\left(p_{i}, t_{O_{j}}\right)$ and $e\left(t_{I_{i}}, p_{i}\right)$ respectively, and $\alpha_{j}$ satisfies $\alpha_{1} \geq \alpha_{2} \geq \cdots \geq \alpha_{n} . t_{I_{i}}, t_{O_{j}}$ are the input and output transitions of place $p_{i}$, and $f_{I_{i}}, f_{O_{j}}$ are the maximum of firing frequencies of $t_{I_{i}}$ and $t_{O_{j}}$, respectively as shown in figure 8 . 
Table 1: Biological interpretation of each transition in figure 5.

\begin{tabular}{|c|c|c|}
\hline Transition & Reaction type & Biological interpretation \\
\hline $\begin{array}{l}t_{1}, t_{3}, t_{5}, t_{7}, t_{16}, t_{17}, t_{20} \\
t_{24}, t_{26}, t_{28}, t_{29}, t_{33}, t_{41}, t_{48}\end{array}$ & XII. Production/Degradation & $\begin{array}{l}\text { The substances represented by output places transitions } \\
\text { attend the reactions from system environment }\end{array}$ \\
\hline$t_{2}, t_{32}, t_{44}$ & VII. Homodimerization & $\begin{array}{l}\text { Polymerization reaction of some identical substances to } \\
\text { shape a multimer }\end{array}$ \\
\hline$t_{4}, t_{6}, t_{8}, t_{23}, t_{34}$ & I. Association & $\begin{array}{l}\text { Association reaction with the binding to induce the for- } \\
\text { mation of a complex }\end{array}$ \\
\hline$t_{9}, t_{35}$ & X. Dissociation & $\begin{array}{l}\text { Complexes, substances separate or split into smaller } \\
\text { molecules }\end{array}$ \\
\hline $\begin{array}{l}t_{10}, t_{12}, t_{14}, t_{15}, t_{38}, t_{39} \\
t_{40}, t_{42}, t_{49}\end{array}$ & XI. Enzymic reaction & $\begin{array}{l}\text { Substrates are catalyzed to the productions; there occurs } \\
\text { no consumption in biochemical reactions }\end{array}$ \\
\hline $\begin{array}{l}t_{11}, t_{13}, t_{18}, t_{30}, t_{31}, t_{36} \\
t_{37}, t_{43}, t_{45}, t_{46}, t_{47}, t_{50}, t_{51}\end{array}$ & XII. Production/Degradation & Natural degradation of substances \\
\hline$t_{19}, t_{22}$ & VIII. Translocation & $\begin{array}{l}\text { The movement action of substances from cytoplasm to } \\
\text { mitochondria membranes }\end{array}$ \\
\hline$t_{21}, t_{25}, t_{27}$ & VI. Chemical reaction & $\begin{array}{l}\text { The conversion of substances to products by a chemical } \\
\text { reaction }\end{array}$ \\
\hline
\end{tabular}

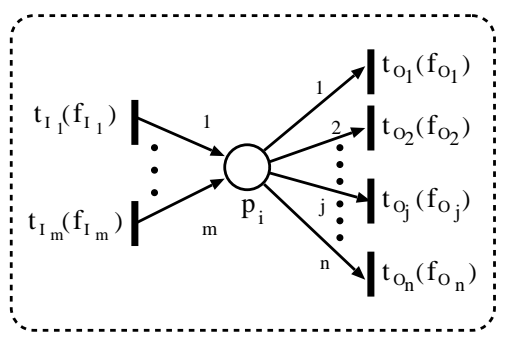

Figure 8: Conflict situation applied to Rule (2).

Rule (3): use a small value as the maximum of firing frequencies to sink transition connected from the enzyme place.

In the above Rule (1), the equation is applied to such a place that has a single output transition. Obviously, this single transition can fire smoothly only according to the delay time. However, in apoptosis Petri net model, there exist the situations that transitions (e.g. $t_{14}, t_{39}$ and $t_{40}$ ) are in conflict since firing either will remove the token from the input place disabling the other transitions. As we have stated above, biological experiments for measuring such reactions have not been executed yet in the majority of cases. Therefore, in this paper we assume such enabled transitions in conflict have the same chance to fire. Under this assumption, the first equation of Rule (2) is designed to obey Basic principle (1), and the second inequality is considered for two reasons that: (i) the right-hand member $\frac{f_{O_{1}}}{\alpha_{1}} \geq \frac{f_{O_{2}}}{\alpha_{2}} \geq \cdots \geq \frac{f_{O_{n}}}{\alpha_{n}}$ is designed to guarantee the firing of $t_{O_{1}}$ with the maximum arc-weight $\alpha_{1}$ that $t_{O_{1}}$ do not fire later than the firing of $t_{O_{n}}$ with minimum arc-weight $\alpha_{n}$; (ii) the left-hand member $2 \cdot \frac{f_{O_{n}}}{\alpha_{n}} \geq \frac{f_{O_{1}}}{\alpha_{1}}$ is in order to make the transition $t_{O_{1}}$ not fire too fast than the $t_{O_{n}}$ that has the minimum arc-weight (make the first firing of $t_{O_{n}}$ earlier than the second firing of $t_{O_{1}}$ to be exactly). Rule (3) is designed to express the moderately slow and small natural degradation of enzymes.

Furthermore, when using timed Petri net to simulate the apoptosis, the self-loops of enzyme places are replaced by test arcs with threshold due to the attribute that a test arc does not consume any content of the place at the source of the arc by firing. The firing rules are defined as follows:

(1) When the value of threshold equals 1 , nothing need to be done since the transition at the sink of test arc can fire constantly as long as the place at the source of test arc is occupied by tokens;

(2) When the value of threshold is more than 1, the test arc is handled as general arc and the transition at its sink has to comply with the above rules in order to guarantee the firing of the transition connected from the test arc.

In the following, we demonstrate how our model is practically executed (simulated). Here, the explanation of several transitions applied to corresponding rules is detailedly given as an example. Furthermore, applying timed Petri net model allows to replace the initial marking instead of the source transitions, because of the facts that survival cells receive a certain number of extracellular stimuli and the amount of substances involved in corresponding reaction is not infinite (but enough many). And the number of initial tokens can be obtained. For example, the number of initial tokens for the place of Fas ligand equals $\alpha \cdot f_{2}=3 \cdot 1=3$ obtained by applying Rule (1) due to its single output transition $t_{2}$. Note that, the delay time of the most apical transition is supposed to 1 .

Figure 9 illustrates the timed Petri net model of apoptosis. In figure 9, the place of Fas ligand trimer is such a place that the number of input and output transitions is respectively one, so that the maximum of firing frequencies $f_{4}$ satisfy the equation of Rule (1), i.e. $\beta \cdot f_{2}=\alpha \cdot f_{4}$, where, since $\beta=\alpha=1$, and $f_{2}=1$, then $f_{4}=1$ is obtained. In calculating the delay time of $t_{9}$ and $t_{10}$, we apply Rule (2) because they are in conflict. Based on the equation of $\beta \cdot f_{8}=\alpha_{1} \cdot f_{9}+\alpha_{2} \cdot f_{10}$, since $\beta=\alpha_{1}=\alpha_{2}=1, f_{8}=1$, and $f_{9}$ and $f_{10}$ are assumed to have the same firing chance, $f_{9}=f_{10}=1 / 2$ are obtained and accordingly $d_{9}=d_{10}=2$ are obtained. That is, the transition $t_{9}$ and $t_{10}$ have the equal chance to fire after $d_{9}$ or $d_{10}$. Note that $\beta$ is the arcweight of $e\left(t_{8}, p_{8}\right) . \alpha_{1}$ and $\alpha_{2}$ are the arc-weight of $e\left(p_{8}, t_{9}\right)$ 


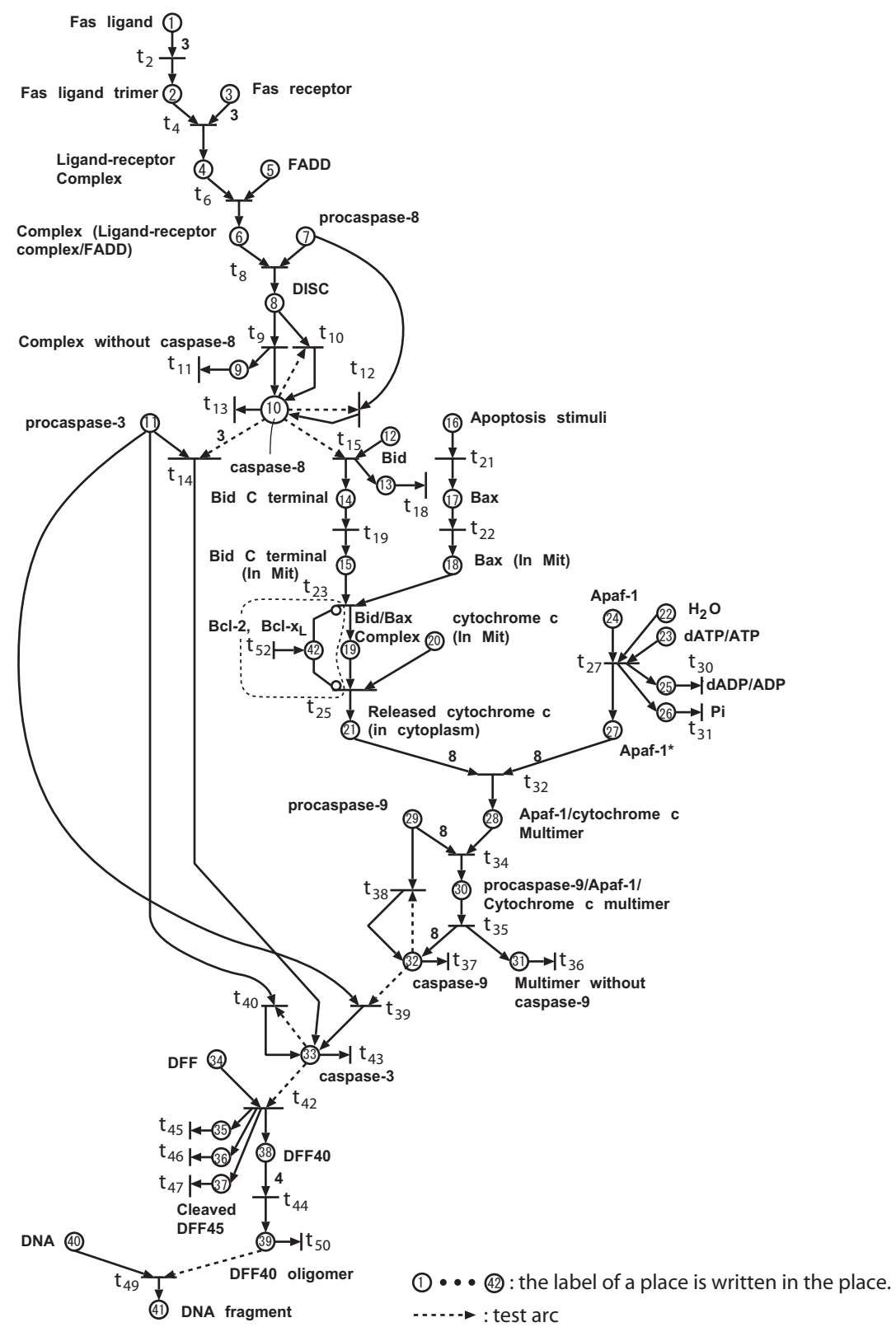

Figure 9: Timed Petri net model of apoptosis based on figure 5. The block surrounded by dashed lines does not take part in the simulation of timed Petri net models because of the assumption that apoptosis pathways propagate the 'signal' without receiving the signals from any signal pathway having a relation to $t_{52}$ when simulating the timed Petri net.

and $e\left(p_{8}, t_{10}\right)$, respectively. The delay time $d_{37}$ of the transition $t_{37}$ is assigned to 10 as an example that is based on Rule (3) due to the feature of small and natural degradation of caspase-9.

\section{Simulation results and discussions}

With the processes to decide the delay time of transitions to timed Petri net model in section 3.2, the delay time for transitions can be determined and the number of initial tokens of places can be given as listed in tables 2 and 3. The constructed timed Petri net model (figure 9) of apoptosis is simulated by using Cell Illustrator with the decided delay time and the number of initial tokens.

In the Petri net model as shown in figure 5, the production of $\mathrm{Bcl}-2 / \mathrm{BCL}-\mathrm{x}_{L}$ is generally influenced by the activities of other signaling pathways, thus the place $p_{42}$ of Bcl-2/BCL$\mathrm{x}_{L}$ and input transition $t_{52}$ are specially adopted to represent these activities. However, the apoptosis pathway of this paper is supposed to propagate the 'signal' without receiving the signals from any signal pathway having a relation to $t_{52}$ when simulating the timed Petri net. That is, the transition $t_{52}$ does not fire. Therefore, the simulation of timed Petri net models can be performed without inhibitor arcs.

The simulation results are presented in figure 10:

(1) The response of DNA fragments to Fas ligands is expressed as the token behaviors in figure 10 (a): Fas ligand concentration reaches zero at the time 2.0 [sec], and DNA fragments that is often considered as an indicator of cell death, start to increase after the time 19.0 [sec]. Apoptotic pathways are working to propagate signals from Fas ligands to the DNA; 
Table 2: The number of initial tokens for the output places of source transitions.

\begin{tabular}{|c||c|c|}
\hline Place $p_{i}$ & Biological substance & Initial tokens \\
\hline \hline$p_{1}$ & Fas ligand & 6 \\
\hline$p_{3}$ & Fas receptor & 6 \\
\hline$p_{5}$ & FADD & 6 \\
\hline$p_{7}$ & procaspase-8 & 7 \\
\hline$p_{11}$ & procaspase-3 & 28 \\
\hline$p_{12}$ & Bid & 8 \\
\hline$p_{16}$ & Apoptosis stimuli & 8 \\
\hline$p_{20}$ & cytochrome c & 8 \\
\hline$p_{22}$ & H 2 O & 8 \\
\hline$p_{23}$ & dATP/ATP & 8 \\
\hline$p_{24}$ & Apaf-1 & 8 \\
\hline$p_{29}$ & procaspase-9 & 9 \\
\hline$p_{34}$ & DFF & 4 \\
\hline$p_{40}$ & DNA & 10 \\
\hline \multicolumn{2}{|c||}{} &
\end{tabular}

Table 3: Transition speeds in timed Petri net model for apoptosis illustrated in figure 9.

\begin{tabular}{|c||c|c||c|c|c|}
\hline Transition $t_{i}$ & Reaction type & Delay time $d_{i}[\mathrm{sec}]$ & Transition $t_{i}$ & Reaction type & Delay time $d_{i}[\mathrm{sec}]$ \\
\hline \hline$t_{2}$ & VII. Homodimerization & 1 & $t_{30}$ & XI. Degradation & 1 \\
\hline$t_{4}$ & I. Association & 1 & $t_{31}$ & XI. Degradation & 1 \\
\hline$t_{6}$ & I. Association & 1 & $t_{32}$ & VII. Homodimerization & 8 \\
\hline$t_{8}$ & I. Association & 1 & $t_{34}$ & I. Association & 8 \\
\hline$t_{9}$ & X. Dissociation & 2 & $t_{35}$ & X. Dissociation & 8 \\
\hline$t_{10}$ & XI. Enzymic reaction & 2 & $t_{36}$ & XI. Degradation & 8 \\
\hline$t_{11}$ & XII. Degradation & 2 & $t_{37}$ & XI. Degradation & 10 \\
\hline$t_{12}$ & XI. Enzymic reaction & 1 & $t_{38}$ & XI. Enzymic reaction & 8 \\
\hline$t_{13}$ & XII. Degradation & 10 & $t_{39}$ & XI. Enzymic reaction & 1.5 \\
\hline$t_{14}$ & XI. Enzymic reaction & 1.5 & $t_{40}$ & XI. Enzymic reaction & 1.5 \\
\hline$t_{15}$ & XI. Enzymic reaction & 1 & $t_{42}$ & XI. Enzymic reaction & 1 \\
\hline$t_{18}$ & XI. Degradation & 1 & $t_{43}$ & XI. Degradation & 10 \\
\hline$t_{19}$ & VII. Translocation & 1 & $t_{45}$ & XI. Degradation & 1 \\
\hline$t_{21}$ & VI. Chemical reaction & 1 & $t_{46}$ & XI. Degradation & 1 \\
\hline$t_{22}$ & VII. Translocation & 1 & $t_{47}$ & XI. Degradation & 1 \\
\hline$t_{23}$ & I. Association & 1 & $t_{49}$ & XI. Enzymic reaction & 1 \\
\hline$t_{25}$ & VI. Chemical reaction & 1 & $t_{50}$ & XI. Degradation & 10 \\
\hline$t_{27}$ & VI. Chemical reaction & 1 & & \\
\hline
\end{tabular}

(2) the diagram (b) shows the number of tokens and further the activation order of three kinds of caspases involved in apoptosis pathways:

(i) caspase- 8 is activated ahead of caspase- 3 and caspase-9;

(ii) caspase-3 is activated afterwards which is activated directly by caspase- 8 and the amount of caspase- 3 has a notable augment that can be observed as the result of the autocatalytic activation;

(iii) caspase- 9 begins to be activated after a short time from the activation of caspase- 3 . This is due to the activation of caspase- 9 which relies on the mitochondrial DNA damage pathways that include the reactions such as the release of cytochrome $\mathrm{c}$ from mitochondria, the formation of apoptosome and so on.
By comparing the behaviors of simulation results executed by Matsuno et al. [16], we can observe that:

(1) both of the diagram (a) and (b) in figure 10 have the approximate same waveforms as the simulation results in Ref. [16], i.e. the amount of DNA destruction is increased along with the decrease of Fas ligands due to the complex formation of Fas ligand trimer with the lapse of time.

(2) the activation order of three kinds of caspases is caspase-8, caspase-3, caspase- 9 , which is exactly the same as the one derived from the simulation in Ref. [16].

Since the simulation results reported by Matsuno et al. [16] have been successfully obtained by using "hybrid Petri nets", our proposed simulation method may probably provide quite promising results based on timed Petri net model. However, hybrid Petri nets use continuous places and transitions. The reaction rates of transitions are assigned as real numbers 


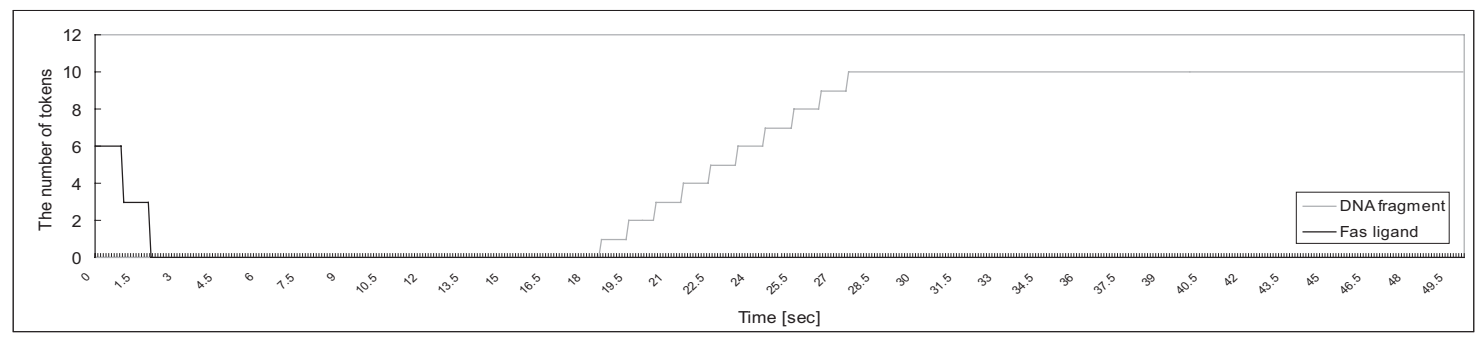

(a) Simulation results of Fas ligand and DNA fragmentation.

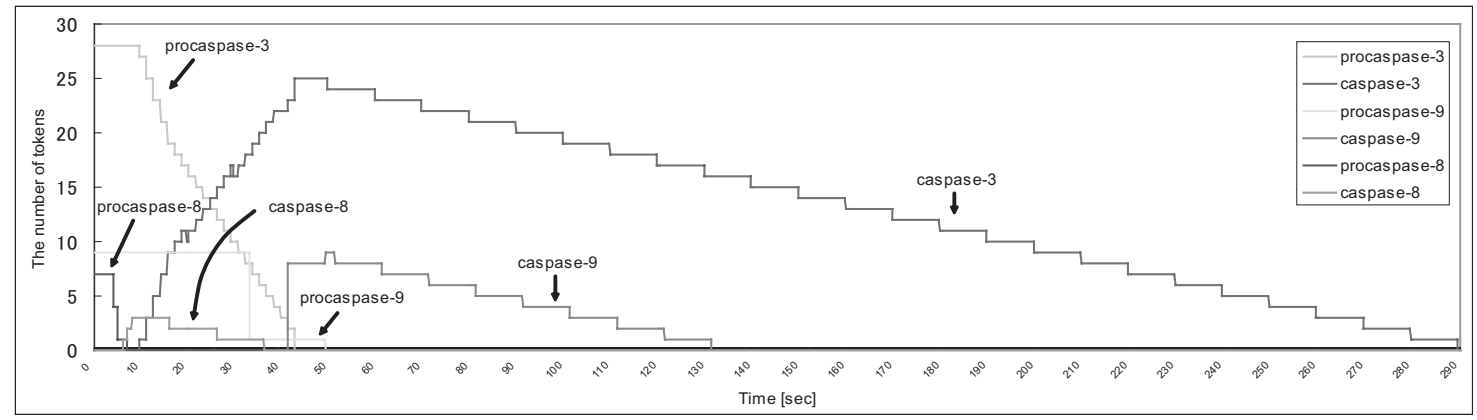

(b) Simulation results of procaspases and caspases.

Figure 10: Simulation results of timed Petri net model of apoptosis in figure 9. (a) Token numbers representing DNA fragments increase in response to the initial amount of tokens of Fas ligands. (b) Concentration behaviors of (pro-)caspases working in the apoptotic pathway. The order of expression of the caspases in this graph is exactly the same as the order of them in Ref. [16], which is obtained by manual tuning of transitions in the apoptotic pathway.

and the parameters for initial concentration of substances and transition speeds are carefully tuned by hand. In general, lots of trail and error processes have been performed until appropriate parameters for simulation are determined. The simulation is precise and is executed in very small time interval. The parameter tuning processes consume too much time. Therefore, with the simulation results, the appropriateness of the timed Petri net model of apoptosis is verified that the simulation method may probably provide a number of valuable insights.

In addition, since Cell Illustrator possesses the excellent graphical user interface, the timed Petri net of apoptosis can be simulated in the animation method. In this way, the different apoptotic pathways can be pursued by tracing the flow of tokens as follows:

(1) Fas ligand induced pathway:

Fas ligand/Fas receptor/FADD/DISC/procaspase-8/ caspase-8/procaspase-3/caspase-3 pathway

(2) Mitochondrial DNA damage pathway:

Fas ligand/Fas receptor/FADD/DISC/procaspase-8/ caspase-8/Bid/Bax/cytochrome c/Apaf-1/apoptosome/ caspase-9/caspase-3 pathway.

In contrast, the continuous Petri net models have no such feature to make the biologists intuitively understand the intrinsic structure and behavioral properties of signaling pathways by simulating the models.

\section{Concluding remarks}

We have first presented basic Petri net components representing molecular interactions of signaling pathways, and in- troduced a method to construct a Petri net model of a signaling pathway with these components. Then we have described a method of determining transition speeds of a Petri net based on some simple principles that the number of tokens flowed into a place is equivalent to the number of tokens flowed out of the place. Finally we have confirmed the availability of proposed method through the modeling and simulation of the apoptosis signaling pathway. The main contributions are that:

(1) to give a consistent description of signaling pathways with the Petri net models of molecular interactions and mechanisms in figure 3, which enables not only biologists but also researchers in computer science and/or engineering to intuitively understand the intrinsic structure and features of signaling pathways;

(2) to propose a procedure to automatically determine flow speeds of different transductions leading intracellular responses to ultimate regulation, which are determined along with the transition speeds of Petri net.

Therefore, the simulation method on timed Petri net model of apoptosis may probably be an available one to provide a number of valuable results. As the future work, the following problems are open to be solved: (i) to simulate other Petri net modeled biological pathways; (ii) to find efficient method for converting timed Petri net model to a continuous one that enables higher predictive precision.

In addition, the website so-called "Petri Net Pathways" (PNP for short) [23] has been built up to open since 1st April, 2006. This website provides biological pathway models with Petri nets as well as corresponding detailed explanation by flash animation that can make the biologists intuitively under- 
stand intrinsic structure and features of biological pathways. Further, by employing the simulation techniques proposed in this paper, it is possible to mechanically simulate timed Petri net models and obtain valuable insights from the results of analyses and simulations. Now, PNP has presented three Petri net models of IL-1, G-protein and TPO signaling pathways. The rest Petri net models for the pathways such as IGF-1, IL3, PDGF and TGF-beta signaling pathways are planned to be added soon.

\section{Acknowledgments}

The authors are grateful to Dr. Atsushi Doi at University of Tokyo for valuable discussions. This work was partially supported by Grant-in-Aid for Scientific Research on Priority Areas "Systems Genomics" from the Ministry of Education, Culture, Sports, Science and Technology of Japan.

\section{Appendix: Abbreviations}

Apaf-1: apoplectic protease activating factor 1

ATP: $\quad$ adenosine triphosphate

Bad: $\quad$ Bcl- $x_{L} / \mathrm{Bcl}-2$ associated death promoter

Bax: $\quad$ Bcl-2 associated $X$ protein

Bcl-2: B-Cell lymphoma 2

Bid: $\quad$ Bcl-2 interacting protein

caspase: cysteine-aspartic-acid-proteases

dATP: desoxyadenosine triphosphate

DED: death effector domain

DFF: DNA fragmentation factor

DFF40: $\quad 40 \mathrm{kDa}$ unit of DFF

DFF45: $45 \mathrm{kDa}$ unit of DFF

DISC: death inducing signaling complex

FADD: Fas-associated death domain protein

tBid: $\quad$ truncated Bid

TNF: tumor necrosis factor

\section{REFERENCES}

[1] Choi C 2004 Novel consistent modeling of signaling pathways and its implementation in the TRANSPATH database; Genome Informatics 15(2) 244-254

[2] Cell Illustrator; http://www.gene-networks.com/

[3] Genrich H 2001 Executable Petri net models for the analysis of metabolic pathways; International Journal on Software Tools for Technology Transfer 3(4) 394-404

[4] Hatakeyama M 2003 A computational model on the modulation of mitogen-activated protein kinase (MAPK) and Akt pathways in heregulin-induced ErbB signalling; Biochem J. 373(2) 451-463

[5] Heiner M 2001 Analysis and simulation of steady states in metabolic pathways with Petri nets; $C P N$ 'O1 - Third Workshop and Tutorial on Practical Use of Coloured Petri Nets and the CPN Tools Jensen K (ed) University of Aarhus, Denmark 15-34

[6] Heiner M 2004 Model validation of biological pathways using Petri nets, demonstrated for apoptosis; Biosystems 75(1-3) 15-28
[7] Hofestädt R 1994 A Petri net application to model metabolic processes; Syst. Anal. Mod. Simul. 16(2) 113122

[8] Hofestädt R 1998 Quantitative modeling of biochemical networks; In Silico Biol. 1(1) 39-53

[9] Hu Y 1999 Role of cytochrome $\mathrm{c}$ and dATP/ATP hydrolysis in Apaf-1-mediated caspase-9 activation and apoptosis; EMBO J. 18(13) 3586-3595

[10] Jacobson M D 1997 Programmed cell death in animal development; Cell 88(3) 347-354

[11] Küffner R 2000 Pathway analysis in metabolic databases via differential metabolic display (DMD); Bioinformatics 16(9) 825-836

[12] Lee D Y 2004 Knowledge representation model for systems-level analysis of signal transduction networks; Genome Informatics 15(2) 234-243

[13] Li P 1997 Cytochrome c and dATP-dependent formation of Apaf-1/caspase-9 complex initiates an apoptotic protease cascade; Cell 91(4) 479-489

[14] Luo X 1998 Bid, a Bcl2 interacting protein, mediates cytochrome $\mathrm{c}$ release from mitochondria in response to activation of cell surface death receptors; Cell 94(4) 481490

[15] Matsuno H 2000 Hybrid Petri net representation of gene regulatory network; Pacific Symp. on Biocomputing 341-352

[16] Matsuno H 2003 Biopathways representation and simulation on hybrid functional Petri net; In Silico Biol. 3(3) 389-404

[17] Matsuno H 2003 Towards biopathway modeling and simulation; Proc. 24th ICATPN (Lecture Notes in Computer Science) 2679 3-22

[18] Matsuno H 2006 Petri net based descriptions for systematic understandings of biological pathways; IEICE Trans. Fundamental (in press)

[19] Nagata S 1997 Apoptosis by death factor; Cell 88(3) 355-365

[20] Narahari Y 1989 Discrete event simulation of distributed systems using stochastic Petri nets; Energy, Electronics, Computers, Communications 622-625

[21] Peccoud J 1998 Stochastic Petri nets for genetic networks; Medicine Sciences 14(8-9) 991-993

[22] Peterson J 1981 Petri net theory and the modeling of systems (Englewood Cliffs, NJ.: Prentice-Hall Inc.)

[23] Petri Net Pathways; http://genome.ib.sci.yamaguchiu.ac.jp/ pnp/

[24] Pinney J 2003 Petri net representations in systems biology; Biochemical Society Trans. 31(6) 1513-1515

[25] Popova-Zeugmann L 2005 Time Petri nets for modelling and analysis of biochemical networks; Fundamenta Informaticae 67(149-162)

[26] Reddy V N 1993 Petri net representations in metabolic pathways; Proc. the 1st Int. Conf. on Intell. Syst. for Mol. Biol. 328-336

[27] Reddy V N 1996 Qualitative analysis of biochemical reaction systems; Comput. Biol. Med 26(1) 9-24 
[28] Saleh A 1999 Cytochrome c and dATP-mediated oligomerization of Apaf-1 is a prerequisite for procaspase-9 activation; J Biol Chem. 274(25) 1794117945

[29] Sasagawa S 2005 Prediction and validation of the distinct dynamics of transient and sustained ERK activation; Nat. Cell Biol 7(4) 365-373

[30] Scaffidi C 1998 Two CD95 (APO-1/Fas) signaling pathways; EMBO J. 17(6) 1675-1687

[31] Schuster S 2000 Structural Analysis of Metabolic Networks: Elementary Flux Mode, Analogy to Petri Nets, and Application to Mycoplasma pneumoniae; German Conference on Bioinformatics 2000 115-120

[32] Takai-Igarashi T 2004 Cell signaling networks ontology; In Silico Biol. 4(1) 81-87

[33] Thompson C 1995 Apoptosis in the pathogenesis and treatment of disease; Science 267(5203) 1456-1462

[34] Voss K 2003 Steady state analysis of metabolic pathways using Petri nets; In Silico Biol. 3(3) 367-387

[35] Weber C H 2001 A docking model of key components of the DISC complex: death domain superfamily interactions redefined; FEBS Lett. 492(3) 171-176

[36] Widlak P 2003 Subunit structures and stoichiometries of human DNA fragmentation factor proteins before and after induction of apoptosis; J Biol Chem. 278(29) 26915-26922

[37] Zevedei-Oancea I 2003 Topological analysis of metabolic networks based on Petri net theory; In Silico Biol. 3(3) 323-345

[38] Zou H 1999 An Apaf-1.Cytochrome c multimeric complex is a functional apoptosome that activates procaspase-9; J Biol Chem. 274(17) 11549-11556 